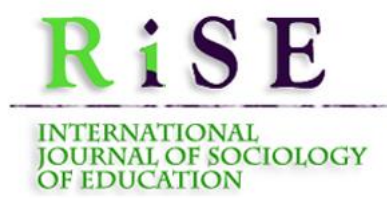

Hipatia Press

www.hipatiapress.com



Instructions for authors, subscriptions and further details:

http://rise.hipatiapress.com

\title{
Aprendizaje Intergeneracional en Contextos Familiares y Socio- Educativos. Estudio de Caso en la Comarca Extremeña de Sierra Suroeste
}

Santiago Cambero Rivero ${ }^{1}$

Noelia Rangel Preciado ${ }^{1}$

1) University of Extremadura

Date of publication: February $25^{\text {th }}, 2020$

Edition period: February 2020-June 2020

To cite this article: Cambero Rivero, S. \& Rangel Preciado, N. (2020). Aprendizaje Intergeneracional en Contextos Familiares y Socio-Educativos. Estudio de Caso en la Comarca Extremeña de Sierra Suroeste, International Journal of Sociology of Education, 9(1), 1-33. doi: 10.17583/rise.2020.4210

To link this article: http://dx.doi.org/10.17583/rise.2020.4210

PLEASE SCROLL DOWN FOR ARTICLE

The terms and conditions of use are related to the Open Journal System and to Creative Commons Attribution License (CC-BY) 


\section{Intergenerational Learning in Family and Socio-Educational Contexts. Case Study in the Extremadura Region of the Sierra Suroeste}

Santiago Cambero Rivero

University of Extremadura
Noelia Rangel Preciado

University of Extremadura

(Received: 25 March 2019; Accepted: 19 September 2019; Published: 25 February 2020)

\section{Abstract}

This research shows the knowledge transfers between people of different ages, through meaningful learning processes of concepts in family and socio-educational contexts, which influence children's socialization. The fieldwork was developed using the survey applied to relatives in three localities of the Extremadura region called Sierra Suroeste. The questionnaire focus on the teaching of concepts related to norms and life skills, in addition to the learning relationships between people of three generations within home. The object of study is the verification of knowledge acquired by children of adult family members involved in their socialization, paying attention to grandparents. Likewise, the participant observation was applied in the implementation of intergenerational program at local level, evidencing the transmission of concepts between people of different ages without family relationship. Thus, it was possible to verify the results of this teaching experience, also attending to aspects related to the promotion of autonomy and active ageing. The data emphasize that the main concepts that these minors learn from their adult would be included in the category of "Ethics and morals", "Experiences", "Environmental Education" and "Sports"; while children learn more from their parents, followed by their grandparents, and finally from their siblings.

Keywords: socialization, informal learning, family life education, intergenerational relationships 


\section{Aprendizaje Intergeneracional en Contextos Familiares y Socio-Educativos. Estudio de Caso en la Comarca Extremeña de Sierra Suroeste}

Santiago Cambero Rivero

University of Extremadura
Noelia Rangel Preciado

University of Extremadura

(Recibido: 25 Marzo 2019; Aceptado: 19 Septiembre 2019; Publicado: 25 Febrero 2020)

\section{Resumen}

Esta investigación muestra las transferencias de conocimiento entre personas de distintas edades, mediante procesos de aprendizaje significativo de conceptos en contextos familiares y socio-educativos que influyen en la socialización infantil. El trabajo empírico se desarrolló mediante encuesta aplicada a familiares en tres localidades de la comarca extremeña Sierra Suroeste. El cuestionario focaliza sobre la enseñanza de conceptos relacionados con normas y habilidades para la vida, además de las relaciones de aprendizaje entre personas de tres generaciones en el hogar. El objeto de estudio es la clasificación de conocimientos adquiridos por los niños de familiares adultos que intervienen en su socialización, prestando especial atención a los abuelos. Igualmente, se realizó observación participante en programas municipales intergeneracionales, evidenciando la transmisión de conceptos entre personas de diferentes edades sin relación familiar. Así, pudo comprobarse los resultados de esta experiencia socio-educativa, atendiendo también a aspectos de promoción de la autonomía y el envejecimiento activo. Los datos subrayan que los principales conceptos aprendidos por los menores de sus adultos se categorizan como "Ética y moral", "Vivencias", "Educación Medioambiental" y "Deportes"; mientras que los niños aprenden espontáneamente más de sus progenitores, seguidos de abuelos, y por último, de hermanos.

Palabras clave: socialización, aprendizaje informal, educación familiar, relaciones intergeneracionales 


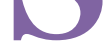

omos parte de sociedades, organizaciones y hogares donde coexisten actualmente hasta cuatro generaciones, generando nuevas pautas residenciales y convivenciales entre personas de distintas edades con vínculos consanguíneos o no. En esta coyuntura sociodemográfico emergen oportunidades para generar otras formas de colaboración y solidaridad intergeneracional que mantengan determinadas condiciones de vida (Mestre et al., 2012). El Estado de Bienestar falla en su función proveedora de servicios públicos, dado su desmantelamiento iniciado hace décadas en Europa, siendo sustituido por las familias como proveedores subsidiarios, principalmente entre las generaciones más jóvenes y mayores. De ahí, como la abuelidad -especialmente las abuelas (Toribio, 2002)- y los roles de los adultos mayores se observan en distintos ámbitos de intervención socio-comunitario (Cambero, 2015; Giró, 2006).

Este trabajo explora y describe las posibilidades de transferencia de conocimiento entre personas de distintas edades y generaciones, a través de procesos de aprendizaje significativo de conceptos en contextos familiares y socio-educativos (no reglados), que influyen en la socialización infantil. También se analiza la influencia de estas enseñanzas desde la perspectiva familiar, especialmente por adultos mayores.

La investigación empírica se efectúo mediante encuesta aplicada a familiares en tres localidades de la comarca extremeña Sierra Suroeste: Oliva de la Frontera, Valencia del Mombuey y Zahínos. El cuestionario focalizó sobre la enseñanza de conceptos relacionados con normas y habilidades para la vida, además de las relaciones de aprendizaje entre familiares de tres generaciones. El objeto de estudio es la comprobación de aquellos conocimientos que adquieren los niños de familiares adultos que intervienen en su socialización, prestando atención a los abuelos como referentes para los menores.

También, se aplicó la observación participante en los programas intergeneracionales organizados entre el Ayuntamiento de Oliva de la Frontera y el CEIP "Maestro Pedro Vera" en Oliva de la Frontera (Badajoz), demostrando la transmisión de conceptos entre personas de diferentes edades y generaciones sin relación de consanguinidad. Se evidenció el impacto individual y colectivo tras esta experiencia socio-educativa en el alumnado de Primaria y los ancianos de pisos tutelados de mencionada localidad, atendiendo igualmente a aspectos sobre la promoción de la autonomía y el 


\section{Cambero \& Rangel-Aprendizaje Intergeneracional}

envejecimiento activo.

Este estudio permite conocer el conjunto de conceptos que los niños aprenden de los distintos miembros de las generaciones intervinientes en su socialización. De los datos obtenidos, subrayar que los principales conceptos aprendidos por estos menores de sus familiares adultos estarían englobados en la categoría de "Ética y moral", "Vivencias", "Educación Medioambiental" y "Deportes"; mientras que los niños aprenden más de sus progenitores, seguidos de sus abuelos, y por último, de sus hermanos.

En conclusión, los conceptos se transmiten principalmente de modo espontáneo, ya que los temas que más se aprenden y transmiten no son los más valorados en la transmisión de conocimiento, sino los que más les gusta tratar a los adultos, es decir, los asuntos relativos a las vivencias personales.

\section{Objetivos de Investigación}

La clasificación de los conceptos adquiridos por los niños mediante la transmisión por miembros de diferentes generaciones intervinientes en la socialización infantil, preferentemente de los abuelos.

Esta investigación estableció los siguientes objetivos secundarios:

1. Identificar las generaciones intervinientes en la socialización y aprendizaje de conceptos de los niños que son objetos de estudio en la comarca extremeña Sierra Suroeste.

2. Conocer los procesos de transmisión intergeneracional de conocimientos.

3. Explorar las relaciones intergeneracionales sin la existencia de lazos familiares.

4. Comprobar si enseñar a otras generaciones beneficia el envejecimiento activo.

En definitiva, evidenciar la trasmisión intergeneracional de conocimiento a través de los talleres observados en los cuales personas mayores muestran sus experiencias a menores. Partiendo de la hipótesis que ambos segmentos de población se benefician recíprocamente de tales experiencias socioeducativas, ya que por un lado, los niños adquieren nuevos conocimientos, y por otro, las personas mayores envejecen activamente mientras cooperan en la socialización infantil. 


\section{Estado de la Cuestión}

El objeto de estudio es la identificación de los actores influyentes en el proceso de enseñanza-aprendizaje del niño, además de analizar los espacios de interacción en los contextos socio-educativo y familiar. Indicar que los sujetos objeto de estudio son niños con edades comprendidas entre 6 y 10 años en las localidades de la mencionada comarca extremeña.

Esta investigación se apoya en el estudio realizado por López Larrosa (2001), pues la mayoría analizan el aprendizaje en contextos familiares y escolares, centrados en temáticas concretas, como el alumnado con necesidades de apoyo específico (Robledo, 2012), la intervención logopédica (Ruíz, 2000) y las habilidades atléticas (Campos, 1998). Otra bibliografía sobre la importancia del aprendizaje de conceptos en el seno familiar para el desarrollo cognitivo y lingüístico del menor fueron Campo (2004), Herranz y Lacasa (1989), McKenna (1980), Moreno (2001), Querejeta (2010) y Vera (1987). Así, con toda esta información se presentan los actores influyentes en el aprendizaje infantil, además de obtener puntos de vista diferentes desde la perspectiva del menor, los progenitores y los adultos mayores.



Figura 1. Actores influyentes en el aprendizaje del niño/a 


\section{Cambero \& Rangel-Aprendizaje Intergeneracional}

En la muestra infantil se comprobó la existencia de tres zonas o grupos de influencia, que intervienen en el aprendizaje de los menores, desde su propia perspectiva. Primeramente, el foco de aprendizaje en la literatura científica es la familia y el hogar, la sociedad y los propios niños, asumiendo que la función social de la educación se desarrolla en la escuela, donde también se ofertan actividades extraescolares. En este entorno hay dos tipos de actores: (1) Docentes y monitores que realizan el rol de enseñantes a los niños. A este grupo no podemos englobarlo dentro de una generación, puesto que en la escuela pública encontramos educadores de edades comprendidas entre 23 y 65 años $^{1}$, y (2) entre iguales, es decir, niños de la misma edad con necesidades de aprendizaje semejantes, aunque de entornos familiares diferentes que generan enseñanza de conceptos de modo espontáneo.

En segundo lugar, el primer núcleo de socialización infantil es el entorno familiar. Dependiendo del modelo familiar existen tres grupos etarios que interactúan en el aprendizaje infantil: (1) Progenitores que ocupan el rol principal en la educación y socialización, que además pertenecen a la misma generación que los tíos; (2) Hermanos y primos que son actores en su aprendizaje que comparten su misma generación aunque su edad puede oscilar, siendo mayores o no al sujeto de estudio, y con más o menos conocimiento; y (3) Abuelos que han adquirido mayor influencia en la educación infantil, ocupándose de su atención ante las ausencias de los progenitores por distintos motivos.

Por último, el entorno exterior del niño, es decir, fuera de la familia y la escuela, en grupos de pares que influyen primariamente en la socialización y aprendizaje: (1) Amistades que no tienen por qué tener la misma edad, lo que implica que si éstas son mayores pueden ser un foco de aprendizaje infantil de más conocimiento; y (2) Resto de actores con los que los niños interactúan, como son el vecindario, las amistades familiares, etc.

Esta tabla agrupa los actores influyentes en el aprendizaje de conocimiento desde la perspectiva generacional. La disciplina sociológica aborda este concepto como polisémico y cambiante temporalmente (Caballero y Baigorri, 2013), aunque cada generación son cohortes de edad en la población distinguidos por grupos etarios en función de diferentes vivencias socioculturales. 
Tabla 1

Generaciones en España

\begin{tabular}{cccc}
\hline Generación & Año de inicio & Año de fin & Edad media \\
\hline Silenciosa & 1914 & 1928 & 94 \\
Franquista & 1929 & 1943 & 79 \\
Baby-Boom & 1944 & 1958 & 64 \\
Generación X & 1959 & 1973 & 49 \\
Millenium & 1974 & 1988 & 34 \\
Z & 1989 & - & 13 \\
\hline
\end{tabular}

Fuente: Caballero y Baigorri $(2013,35)$

Esta investigación toma como muestra a los progenitores, abuelos y niños, lo que lleva a conocer a las tres generaciones descritas. Los abuelos con edades comprendidas en dos generaciones como son la Baby-Boom y la franquista, habiendo vivido unos cambios sociales que produjeron cambios axiológicos. A esta generación también se le llama "generación de padres obedientes" debido a la permisividad hacia sus hijos, mientras que la generación franquista exclusivamente en España engloba al grupo etario que conocieron la guerra y postguerra, y con características similares a la "generación silenciosa".

En cuanto a los progenitores se englobarían en la generación millennials o "generación Y". Sus peculiaridades es que fueron educados analógicamente para una sociedad digital, presentando cierto desinterés por asuntos políticos y religiosos. El retraso en la edad de maternidad y paternidad, unido al envejecimiento demográfico en países avanzados, como el caso de España, conlleva que algunos progenitores se encuentren dentro de la "generación X", con características similares a la generación descrita. Por último, los niños se englobarían en la "generación $Z$ " que reciben una educación digital para utilizar las TIC como nativos digitales (Prensky, 2001).

Tratando de generaciones y de las relaciones entre personas de distintas cohortes de edad en las organizaciones que componen la sociedad, se introduce la noción de solidaridad intergeneracional, que fue acuñada en la Segunda Asamblea Mundial sobre el Envejecimiento celebrada en Madrid en el año 2002, definiendo esta solidaridad entre las generaciones a todos los niveles -las familias, las comunidades y las naciones- como fundamental para el logro de una sociedad para todas las edades (Naciones Unidas, 2002). Una sociedad para todas las edades es aquella que ajusta sus estructuras y 


\section{Cambero \& Rangel-Aprendizaje Intergeneracional}

funcionamiento, sus políticas y planes a las necesidades y capacidades de todos, aprovechando por tanto sus posibilidades para beneficio propio, con el objetivo de fortalecer la solidaridad mediante los principios de equidad y reciprocidad entre generaciones (Naciones Unidas, 2002). De ahí la importancia de favorecer iniciativas que sensibilicen socialmente sobre el potencial de las personas jóvenes y mayores, de aunar talento junior y sénior para crear la huella generacional en las organizaciones (Cambero, 2018). Como apuntara Delors (1996), la solidaridad intergeneracional está estrechamente ligada con el aprendizaje a lo largo de la vida y el desarrollo de competencias clave que nos permiten aprender a conocer, aprender a hacer, aprender a ser y aprender a convivir juntos.

A partir de estos objetivos globales, los programas intergeneracionales son vehículos adecuados para el intercambio continuado de recursos y aprendizaje entre las generaciones más viejas y más jóvenes que logren beneficios individuales y sociales (McCrea, Weissman y Thorpe-Brown, 2004). Según el Centro del Conocimiento de Fundación EDE (2015), los programas intergeneracionales posibilitan experiencias de relación y cooperación entre personas de diferentes edades, orientadas a favorecer la transmisión e intercambio de conocimiento, competencias y valores, contribuyendo así a la cohesión y desarrollo comunitarios. De modo que los programas intergeneracionales proporcionan oportunidades a las personas, las familias y las comunidades para disfrutar y beneficiarse de la riqueza de una sociedad para todas las edades (Sánchez, 2007).

La necesidad social de implementar programas intergeneracionales ofrece a todos los grupos etarios determinados roles significativos que implican a individuos, instituciones y organizaciones en acuerdos de colaboración que presten servicio a distintos grupos de edad en sus esfuerzos para ocuparse de necesidades comunitarias tales como el cuidado de niños tras el horario escolar, la seguridad de los barrios o el apoyo de los mayores en soledad (Sánchez, Kaplan y Sáez, 2010).

Entre las prácticas intergeneracionales pueden observarse múltiples formas de interacción, intercambio y cooperación entre generaciones, que favorecen los procesos de aprendizaje-enseñanza entre personas con distintas experiencias vitales cuyos efectos se trasladan a la ciudadanía en general. Los programas intergeneracionales posibilitan cerrar la brecha generacional con beneficios para las generaciones participantes. Las conexiones y las 
relaciones interpersonales, al margen de la edad, es el resultado del compromiso social adquirido por personas jóvenes y mayores (CENIE, 2019).

En resumen, esta investigación analiza descriptiva y explicativamente los resultados del aprendizaje intergeneracional que intervienen en la socialización de la "generación Z", en concreto, en el medio rural de una comunidad como Extremadura, donde se pretende alcanzar una sociedad más inclusiva y cohesionada para todas las edades.

\section{Diseño Metodológico}

Esta investigación recurre a la estadística descriptiva para comparar los resultados tras la aplicación de cuestionario entre las familias de la comarca extremeña de Sierra Suroeste, concretamente en Oliva de la Frontera, Valencia del Mombuey y Zahínos (provincia de Badajoz).

Para la aplicación de la estadística descriptiva se elaboró un cuestionario cerrado (Anexo 1), que se aplicó al encuestado mediante una serie de preguntas, así como la explicación de cada uno de los valores mostrados como respuestas, lo que permitió anotaciones adicionales, es decir, información adicional. Con los resultados obtenidos se compone una base de datos disponible. Y por último, con la finalidad de expresar los resultados se diseñan unas gráficas, extraídos mediante hoja de cálculo.

\section{Población y Muestra}

Características de la población objeto de estudio. Las localidades analizadas poseen una estructura de población dominada por la clase social media y baja, inmersos en un ambiente rural y con influencia cultural por proximidad geográfica a Andalucía y Portugal.

Las características de las generaciones analizadas son las siguientes:

1. La primera generación (denominado "hijos") compuesta por menores entre 6 y 10 años, niños escolarizados que cursan estudios primarios en cada localidad.

2. La segunda generación (denominado "padres/madres") compuesta por adultos con edades comprendidas entre 26 y 42 años, mayoritariamente con estudios primarios y/o secundarios. 


\section{Cambero \& Rangel-Aprendizaje Intergeneracional}

3. La tercera generación (denominados “abuelos") compuestas por adultos mayores de 52 años, entre los que destaca la población analfabeta o que simplemente sabe leer y escribir sin ningún título académico.

Dada la diversidad de modelos familiares, esta investigación se centra en hogares estructurados con progenitores implicados en la educación de sus hijos, tomando como referencia la familia directa del niño.

Elección de la muestra. La elección de la muestra objeto de estudio se ajusta a tres preceptos:

1. Proporcionalidad: Extrapolación de los resultados obtenidos al conjunto de la población de las tres localidades seleccionadas (Oliva de la Frontera, Valencia del Mombuey y Zahínos), de modo que el número de encuestas familiares realizadas se pondera para que sea proporcional a la población de cada una de estas localidades. Se tiene en cuenta el dato disponible el día 1 de enero de 2014 del Instituto Nacional de Estadística.

2. Representatividad: Análisis de tres sectores de la población en base al criterio de pertenencia a distintos grupos etarios y cohortes en las mencionadas localidades extremeñas. En cada una de las encuestas familiares participa un miembro de cada generación descrita, o lo que es lo mismo, el niño, uno de sus progenitores y uno de sus abuelos. Con ello se pretende garantizar la ausencia de sesgo en la muestra de cada generación con el mismo peso de representatividad para el trabajo empírico.

3. Suficiencia: Para la fiabilidad de las encuestas y validez de los datos se deciden que son 36 miembros. Con este número de encuestas garantizamos más de 30 muestras, que hace que los resultados se ajusten a una distribución normal $(0,1)$, al actuar la teoría de los grandes números. 
Tabla 2

Estructura de la población y del muestreo

\begin{tabular}{|c|c|c|c|c|}
\hline Localidad & Población ${ }^{(1)}$ & $\%$ sobre total & Encuestas ${ }^{(2)}$ & $\%$ sobre total ${ }^{(3)}$ \\
\hline Oliva de la Frontera & 5.461 & 60 & $\begin{array}{l}7 \text { familias } \\
\text { (21 miembros) }\end{array}$ & $58 \approx 60$ \\
\hline Valencia del Mombuey & 768 & 8,50 & $\begin{array}{l}1 \text { familia } \\
\text { (3 miembros) }\end{array}$ & 8.50 \\
\hline Zahínos & 2.864 & 31,50 & $\begin{array}{l}4 \text { familias } \\
\text { (12 miembros) }\end{array}$ & $33,5 \approx 31,50$ \\
\hline Total & 9.093 & 100 & $\begin{array}{l}12 \text { familias } \\
\text { (36 miembros) }\end{array}$ & 100 \\
\hline
\end{tabular}

Nota:

(1) Población del término municipal a 1 de enero de 2016.

(2) Se aplica la encuesta a tres miembros de cada familia: Niño/a, padre/madre y abuelo/a.

(3) Se realiza una aproximación porcentual mediante las encuestas realizadas al total de la población.

Fuente: Padrón continuo del INE (consultado el 4 de diciembre de 2016)

\section{Construcción y Aplicación del Cuestionario}

Para la correcta construcción del cuestionario se cumplen los siguientes criterios:

1. Claridad de conceptos, realizando las preguntas de modo compresibles por los miembros de las tres generaciones analizadas, mediante un lenguaje sencillo y directo.

2. Dinámico, haciendo que el cuestionario ocupe unas dimensiones que permita que los encuestados no caigan en aburrimiento ni conteste de forma aleatoria, limitando así las preguntas a trece.

3. Facilitar la comparación, ofreciendo en respuestas similares para diferentes preguntas de forma que nos permitan comparar unas respuestas con otras.

4. Sintético, sintetizado las respuestas a las preguntas mediante el establecimiento de categorías.

5. Abierta, estableciendo unas preguntas fijas para las familias, después de que contesten las mismas el entrevistador podrá preguntar sobre ellas con 


\section{Cambero \& Rangel-Aprendizaje Intergeneracional}

la finalidad de saber cuál es la forma por la cual se transmiten estos conceptos.

Para ello se realizaron preguntas sencillas con respuestas de afirmación o negación. Por ejemplo: Cuándo tu hijo te enseño a reciclar, ¿realizó algún tipo de dibujo o esquema para explicarlo?

En la configuración de las respuestas al cuestionario se distinguen entre dos grupos. En primer lugar, los asuntos y los conceptos que se tratan comúnmente en la familia como deportes o música. Y en segundo lugar, se añaden variables de control, como por ejemplo "Ética". También hay preguntas al final del cuestionario con la finalidad de saber si el sujeto se interesa o no cuando sus familiares intentan enseñarle algo. Del mismo modo también hay preguntas para concretar si las enseñanzas que hace el sujeto a las familias son escuchadas o no.

Por último, para garantizar la realización correcta del cuestionario se ha pasado dicho cuestionario a algunos sujetos del estudio a modo de entrevista, siendo el encuestador el responsable de rellenar el cuestionario a medida que recibía las respuestas del encuestado.

\section{Las Enseñanzas Intergeneracionales en los Hogares Familiares. Interpretación de Resultados.}

En este epígrafe se interpretan los resultados de las encuestas aplicadas a las familias seleccionadas en cada localidad.

Pregunta 1: ¿Sobre qué tema te gusta más hablar cuando estás en familia?

Se analizan aquellos asuntos de conversación que le gustaría tratar a cada uno de los miembros familiares, haciéndolo extensivo a los abuelos que conviven o no en el mismo hogar.

Con esta pregunta se encuentran las familias que les gustaría tratar 10 temas diferentes, siendo los niños los que más temas les gusta abordar, mientras los progenitores y los abuelos los que menor número les gusta abordar. Se entiende que esto puede atender a la hipótesis de que en la etapa infantil se tiene mayor curiosidad por aquellos asuntos que a los familiares adultos no les interesa por su frecuencia (Malrieu, 1979).

En cuanto a la temática más gustada familiarmente son las vivencias, es decir, compartir las experiencias personales de los familiares, probablemente para recibir consejos u orientaciones vitales. Esta categoría también es la 
"Moda" para los progenitores y los abuelos, mientras que para los niños el tema preferente es "Deportes", ambas categorías junto con "Televisión" son las que presentan mayor número de respuestas.

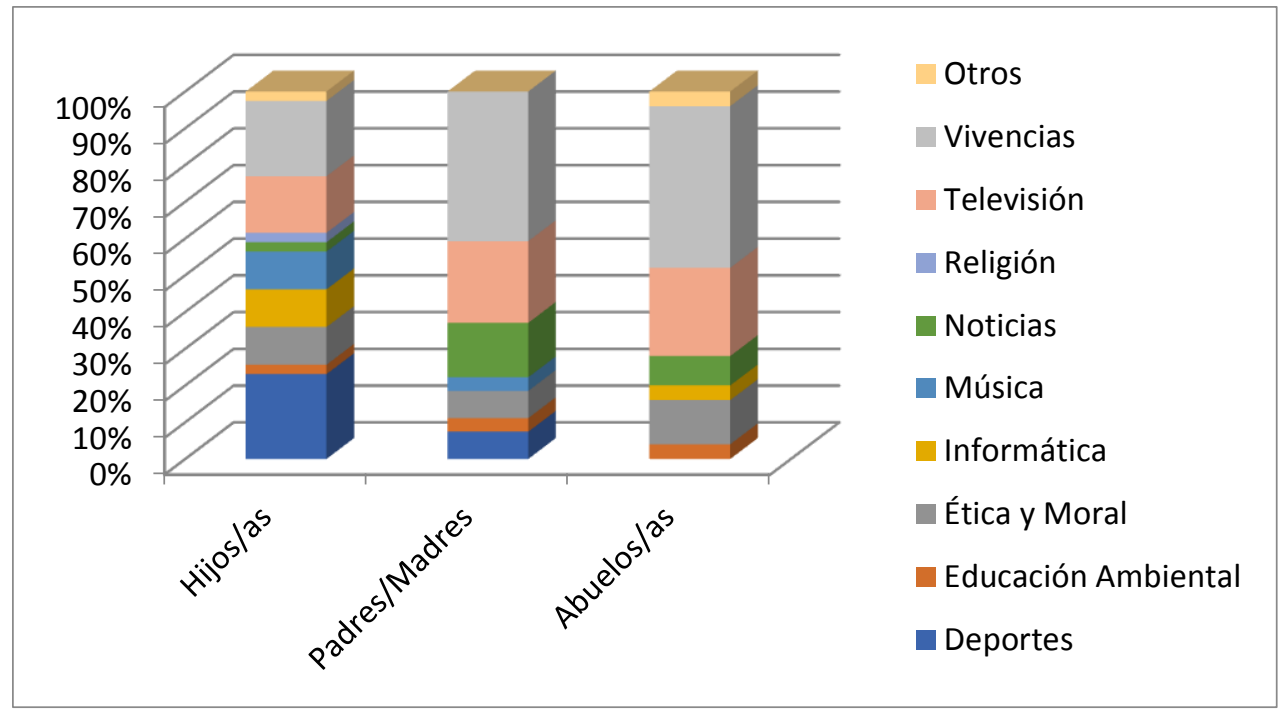

Figura 1. Temas que más gusta tratar en el seno familiar (porcentajes)

Señalar que en el apartado "Otros" una niña ha contestado que lo que más gusta hablar es de "Moda", mientras que una abuela contesta "Costura", al ser ambas de la misma familia, suponemos que es un tema tratado entre ambas.

Pregunta 2: ¿Qué temas tratan en tu familia cuando estáis juntos?

Se encuentra los hogares familiares donde se abordan 11 temas diferentes, siendo los niños los que nuevamente les gusta abordar más asuntos, y por el contrario, siendo tanto los abuelos los que menos temas le gustan.

En cuanto a la temática más tratada en el ámbito familiar son las vivencias, y esta premisa se cumple para todos los miembros, por tanto, y teniendo en cuenta la pregunta anterior, se puede afirmar que se imponen las conversaciones que los mayores quieren tener habitualmente. Es decir, los temas de conversación familiar están más guiados por los adultos mayores que por los menores. 


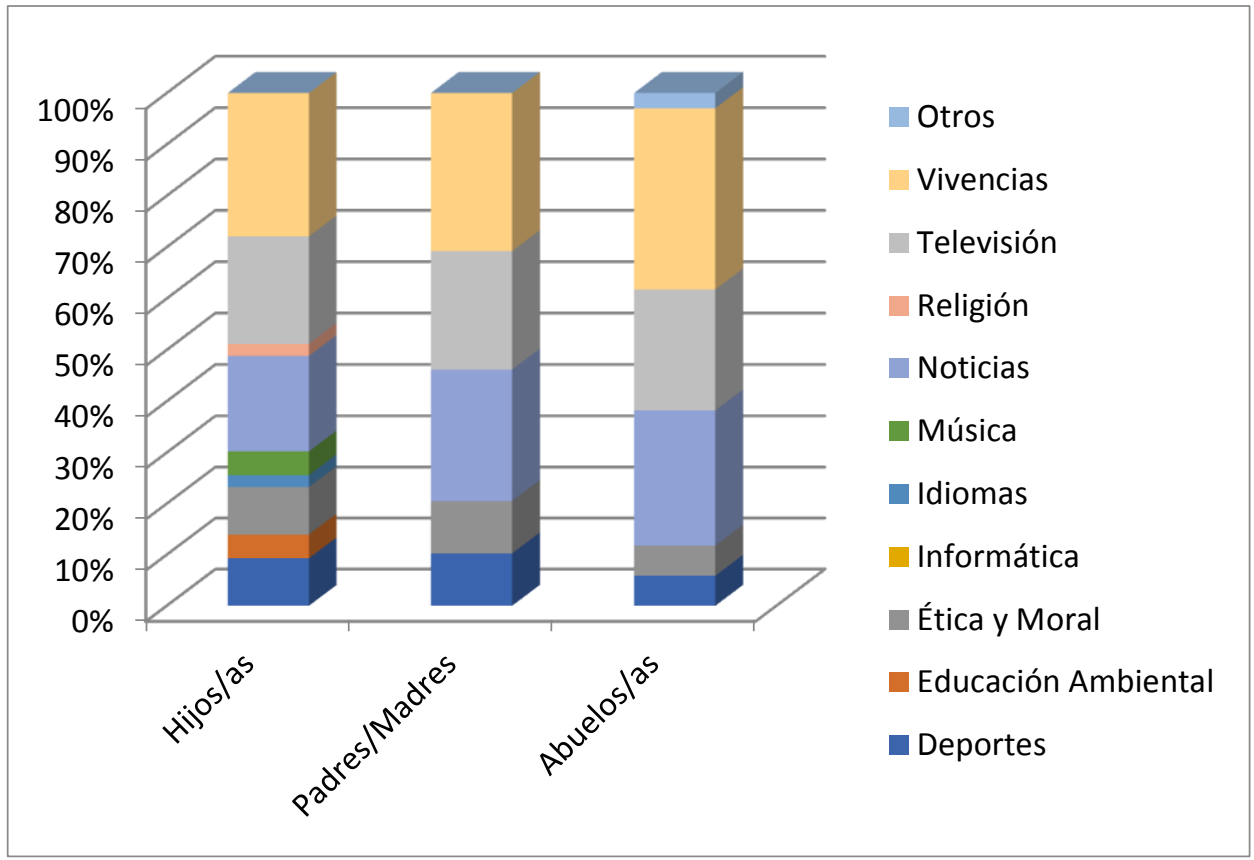

Figura 2. Temas que más gusta tratar en el seno familiar (porcentajes)

Pregunta 3: ¿Cuál son los conceptos que crees que debe enseñar la familia?

Sorprende que la temática más tratada sean las vivencias, que no se corresponda con lo que se considera por sus miembros que debería enseñarse en la familia, pues se entiende que lo relacionado con los valores sociales incluidos dentro de la categoría "Ética y Moral", sean mejor valorados por todos los miembros de la familia.

Aún sorprende más que la "Educación Ambiental", que pueda resultar aparentemente un asunto de menor importancia familiar, tenga importancia en la enseñanza entre generaciones. Por tanto, se puede afirmar que valorando las enseñanzas familiares aparecen la "Ética y Moral" y la "Educación Ambiental", que aun no siendo muy tratados, se entienden que sean necesarios en el aprendizaje familiar. 


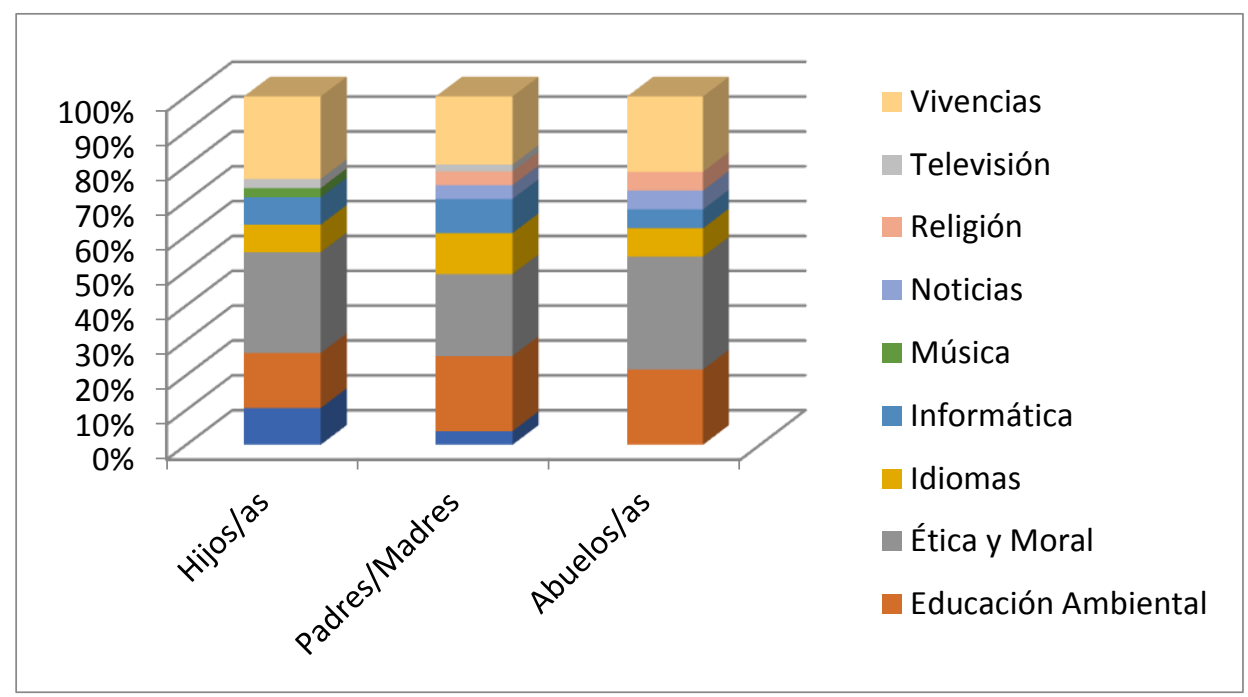

Figura 3. Los conceptos que deben enseñarse (porcentajes)

Pregunta 4: ¿Qué conceptos necesitas que te ayuden a aprender?

Una vez conocidas las temáticas más valoradas por las familias, esta pregunta incide en las necesidades de aprendizaje de cada miembro familiar. Así, el asunto más demandado sería la "Informática", impulsada por la necesidad de progenitores y abuelos por aproximarse a las TIC; mientras que los niños no muestran tal necesidad de aprendizaje y se decantan por los "Idiomas". Este aspecto podría deberse a:

1. En el apartado 2 se comentó que los abuelos y los progenitores fueron formados analógicamente cuando las TIC no tenían tanto impacto, y que hoy resultan desconocidas o nuevas para esas diferentes generaciones. Mientras que los niños son educados en contenidos multimedia desde Internet, propicia que sean quienes puedan enseñar, por ejemplo, el uso de telefonía móvil y otros gadgets electrónicos a adultos mayores.

2. La necesidad de los niños respecto a los idiomas puede resultar una exigencia curricular (Campo, 2004), que cada día se enseña con un carácter instrumental para impartir otras materias en lenguas extranjeras. También destacar la ubicación de los hogares familiares consultados en poblaciones rayanas o fronterizas, suponiendo así una mayor necesidad entre los niños para el aprendizaje del portugués. 


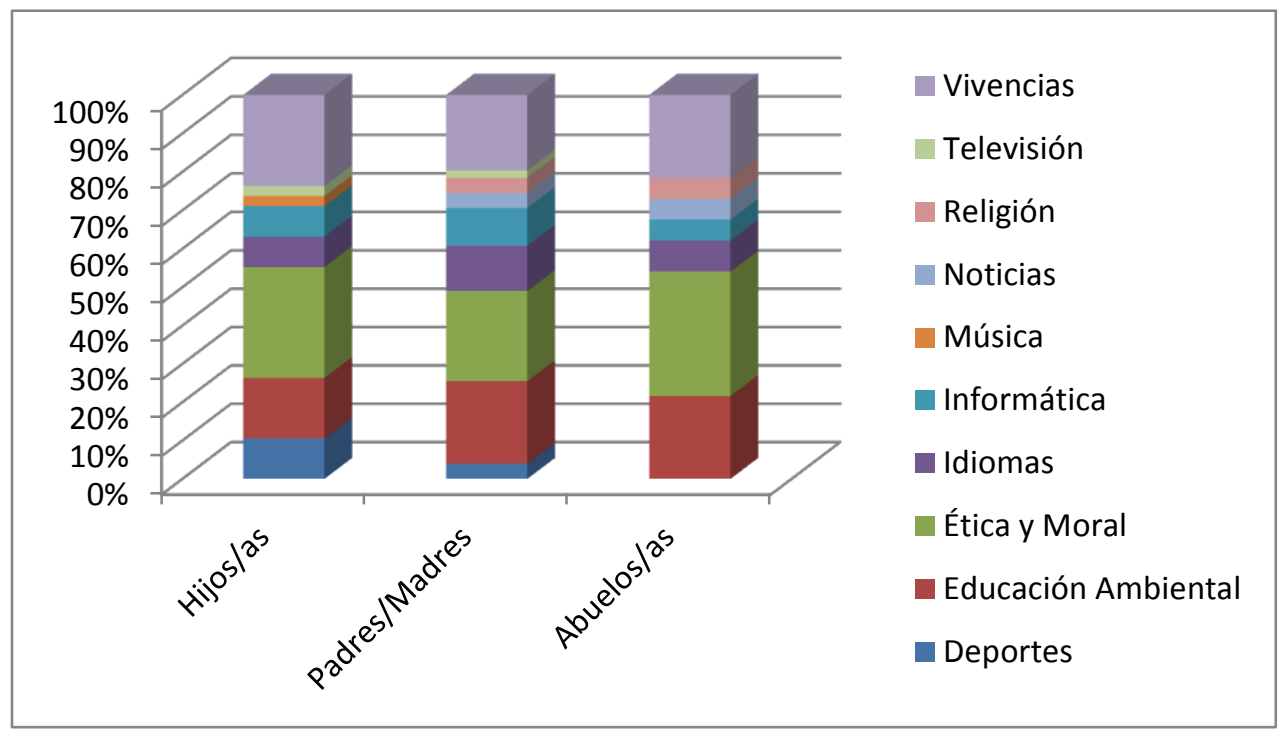

Figura 4. Los conceptos que necesitas aprender (porcentajes)

Pregunta 5: ¿Sobre qué temas tu familia necesita aprender más?

$\mathrm{Al}$ introducir esta pregunta se quería comprobar si la visión de necesidad de aprendizaje que sienten cada uno de los miembros es ratificada por los demás. En el conjunto familiar la "Educación ambiental" es el conocimiento que más debería enseñarse, según datos obtenidos entre progenitores y abuelos; mientras que en el caso de los niños son los deportes lo que más necesitaría aprenderse familiarmente. Curiosamente, el resto de generaciones no los ha tenido en cuenta en esta pregunta, lo que lleva a pensar que quizás los niños consideran que los demás necesitan aprender de deporte porque es la conversación que más le gusta tener. 


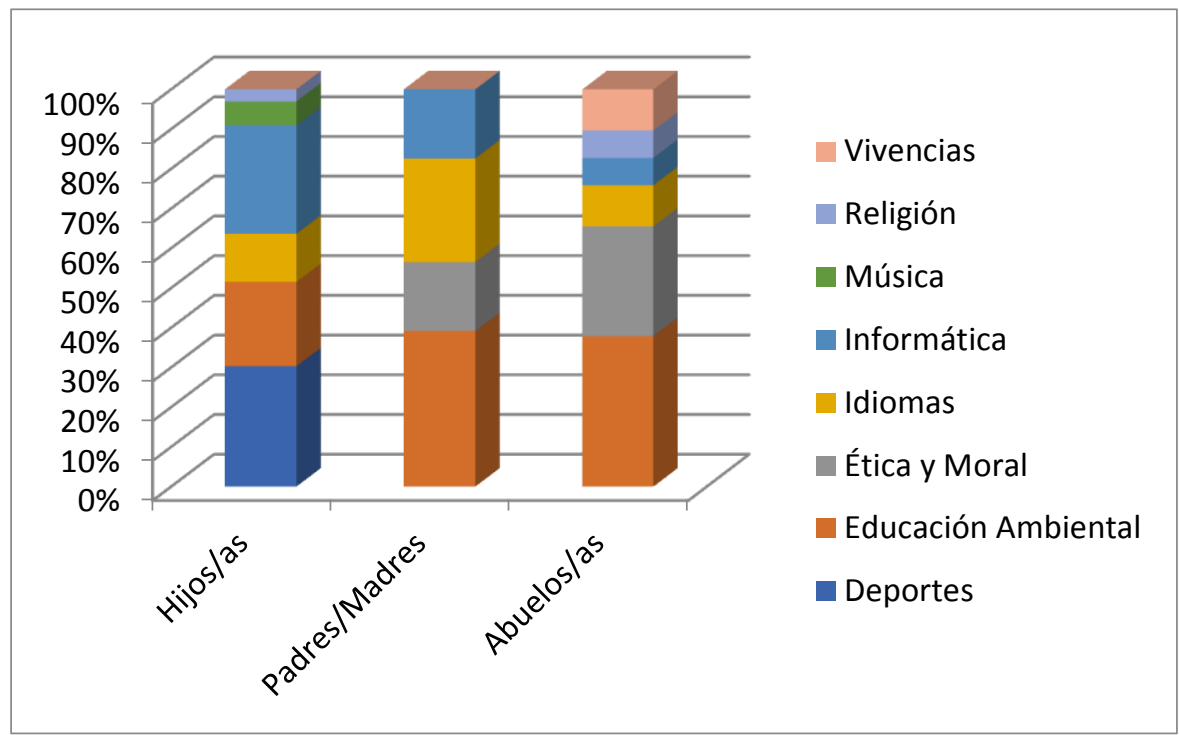

Figura 5. Los conceptos que necesita aprender tu familia (porcentajes)

Pregunta 6: ¿Tu familia te enseño alguno de estos conceptos?

Esta cuestión evidencia aquellos conceptos aprendidos en el hogar familiar, obteniendo respuestas dispares según los grupos de edad y generaciones, resumidos en los siguientes:

1. Deportes: Son los padres y las madres los que más han aprendido, presumiblemente de sus hijos, ya que son los que les gustan estos temas y los que más necesidad reconocen entre los adultos mayores para su práctica física-deportiva.

2. Ética y Moral: Son los niños principalmente los que más aprendan estos conceptos por parte de sus familiares adultos, quienes tienen la misión de inculcar actitudes y valores entre los menores como parte esencial de la socialización.

3. Informática: Son los abuelos los que más han aprendido, comprobando que se refieren a la alfabetización digital requerida para el manejo de la televisión o el teléfono móvil.

Hay otros dos conceptos que también destacan por su importancia para el aprendizaje intergeneracional en los hogares, como:

1. Educación Ambiental: Principalmente entre los niños, como en el caso 


\section{Cambero \& Rangel-Aprendizaje Intergeneracional}

de la "Ética y moral" relacionado con los valores que los adultos enseñan a los menores como parte de su formación integral. Recordar que las familias consultadas habitan en poblaciones del medio rural donde la dehesa tiene funcionalidad social, productiva y ecológica, que genera la transmisión intergeneracional de actitudes de respeto y conservación de este ecosistema extremeño.

2. Vivencias: Cuanto más jóvenes son las generaciones más conceptos habrán aprendido en el hogar familiar sobre vivencias personales de los más añosos, puesto que a mayor edad más experiencias que compartir con personas menores. Este hecho es connatural con la función de la familia como agente de socialización donde conviven personas de distintas edades y generaciones, y generalmente el intercambio se produce en un único sentido por razón de cohorte entre educadores y educandos.



Figura 6. Conceptos aprendidos de la familia (porcentajes) 
Pregunta 7: ¿Las enseñanzas de tu familia son imprescindibles en tu vida?

Esta cuestión resulta importante para valorar el grado de importancia de las enseñanzas familiares, comprobando que la respuesta es unánimemente afirmativa con un $97 \%$, y con un $3 \%$ de respuestas "No sé" de algunos niños.



Figura 7. La imprescindibilidad de las enseñanzas de tu familia

Pregunta 8: ¿Consideras que tus enseñanzas son fundamentales en tu familia?

Al igual que la pregunta anterior, se entendía que las enseñanzas familiares son importantes para la cohesión grupal siendo la respuesta mayoritaria "Sí". 
20 Cambero \& Rangel-Aprendizaje Intergeneracional



Figura 8. La importancia de tus enseñanzas en la familia

Pregunta 9: ¿Quién enseña más en tu familia?

Esta cuestión demuestra que los actores principales son la generación categorizada como padres y madres, mientras que estos aprenden por igual de las otras dos generaciones.

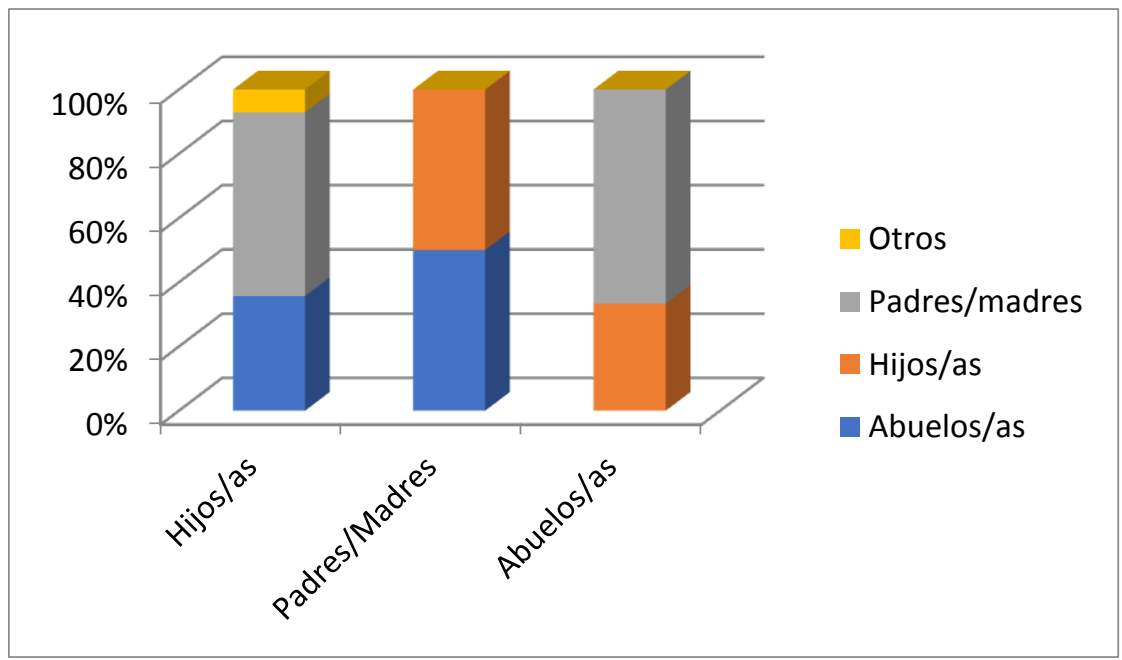

Figura 9. La generación que más enseña en las familias. En porcentaje 
Pregunta 10: ¿A quién te gusta más enseñar en tu familia?

Cuando se preguntó a quién le gusta más enseñar, en este caso los actores principales son los niños. En el siguiente gráfico se observan las respuestas obtenidas donde el círculo que representa cada uno de los valores toma mayor o menor dimensión dependiendo de la importancia de los resultados, y lo mismo ocurre con las flechas a mayor número de respuestas positivas, mayor será su grosor.

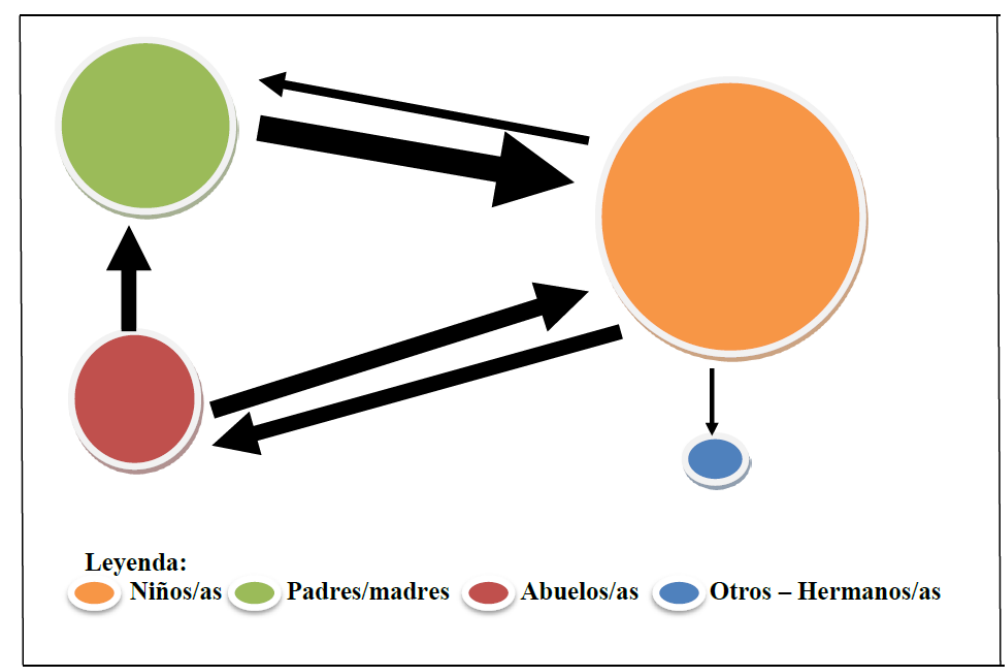

Figura 10. Relaciones de enseñanza en la familia. ¿A quién te gusta más enseñar?

Nota: La dimensión del círculo y anchura de la fecha dependerá del número de veces que se haya repetido la variable analizada

Pregunta 11: ¿A quién le prestas más atención cuando te enseña?

En este caso sobre el miembro familiar al que le prestan más atención cuando enseñan también suelen ser los menores de edad, por razones obvias, aunque solo que aquí las diferencias entre las respuestas tienen valores muy similares para todas las generaciones analizadas. 


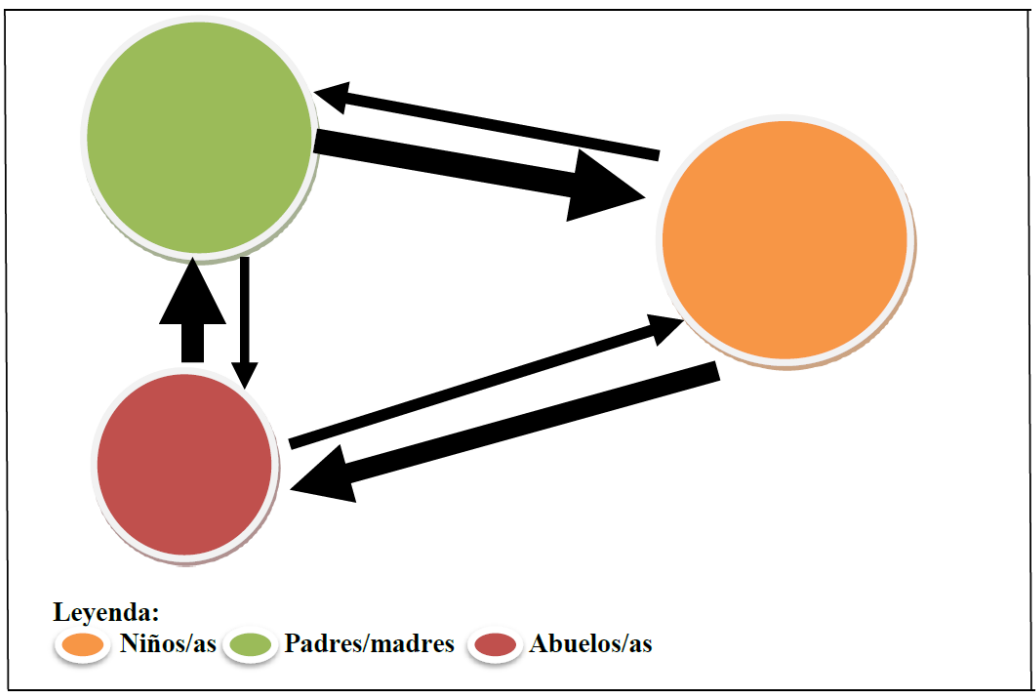

Figura 11. Relaciones de enseñanza en la familia. ¿A quién le prestas más atención cuando te enseña?

Nota: La dimensión del círculo y anchura de la fecha depende del número de veces que se repita la variable analizada

Pregunta 12: ¿Quién te presta más atención cuando tú quieres enseñar algo?

Los valores más altos son para los niños, y cuanto más mayor son las generaciones menos atención se les presta a las enseñanzas de los demás. 




Figura 12. Relaciones de enseñanza en la familia. ¿Quién te presta más atención cuando tú quieres enseñar algo?

Nota: La dimensión del círculo y anchura de la fecha dependerá del número de veces que se repita la variable analizada

Pregunta 13: ¿Te interesa cuando alguien intenta enseñarte?

Por último, el interés que muestra cada uno de los miembros cuando alguien en la familia intenta enseñarle, obteniendo una mayoría de respuestas afirmativas del $92 \%$, el $8 \%$ de las respuestas que solo "a veces" corresponden todas al segmento de niños. 




Figura 13. La importancia de tus enseñanzas en la familia

\section{Experiencias de Enseñanzas Intergeneracionales}

Este epígrafe analiza una experiencia docente desarrollada en el CEIP "Maestro Pedro Vera" durante el curso 2016/2017 y que se repitió en el curso 2017/2018, dado el éxito de participación aunque con una programación mejorada tras la primera experiencia.

Esta acción educativa intencional, debidamente planificada en base al Aprendizaje-Servicio (ApS) como metodología innovadora que pretende modificar la realidad y mejorar los aprendizajes del alumnado, partió de la necesidad del aprendizaje intergeneracional entre personas de la población infantil y sénior. Para ello, se organizó en mencionado colegio un programa de talleres colaborativos con el Ayuntamiento de Oliva de la Frontera que potenciaran la transmisión intergeneracional de conocimiento y experiencias de vida.

\section{Pilotaje del Programa Intergeneracional}

El primer programa intergeneracional desarrollado en el curso académico 2016/2017, participó alumnado de primero y segundo de Primaria con edades comprendidas entre 6 y 8 años. Estos niños interactuaron con los ancianos institucionalizados en los pisos tutelados de la localidad de gestión municipal. Además, aprovechando que se impartía una Escuela-Taller de 
Cocina con alumnado con edades comprendidas entre 18 y 25 años, los organizadores decidieron de forma paralela mostrar a los niños las características de desayunos y almuerzos saludables. De modo que participaban personas de tres generaciones (generación silenciosa, millennials y centennials).

Esta iniciativa socio-educativa tenía como objetivos:

1. Fomentar el aprendizaje de conceptos entre diferentes generaciones, principalmente para que los niños conocieran el estilo de la vida de los ancianos cuando tenían su edad.

2. Promover actitudes de respeto y solidaridad entre personas de distintas edades y generaciones en la localidad, sin vínculo familiar.

3. Incentivar hábitos de vida saludable entre los menores con la alimentación sana y la realización de actividades físico-deportivas.

Los talleres se realizaron en un ambiente lúdico al margen de la dinámica escolar, para que el alumnado se motivara en el aprendizaje intergernacional, con la siguiente programación:

1. Los niños enseñaron sus juegos infantiles y mostraron con dibujos los protagonistas de sus series favoritas. Esta primera actividad estaba dirigida a una primera toma de contacto entre ambos grupos etarios.

2. Los mayores relataron al alumnado sus juegos populares y las vivencias de niñez. Esta actividad tuvo como objetivo que los mayores ejercitaran la memoria con recuerdos, a la vez que el alumnado aprendiera la historia de costumbres locales en otras épocas.

3. Se realizaron actividades deportivas en la piscina cubierta dirigida únicamente a los niños. Esta actividad fomentaba el ejercicio físicodeportivo.

4. El alumnado de la Escuela-Taller de cocina prepararon un desayuno saludable para los niños y los mayores que participaron haciendo dulces. El objetivo principal fueron las prácticas saludables en alimentación, además de reconocer los consejos familiares sobre la alimentación nutritiva expuestos por profesionales.

5. Posteriormente, se organizaron talleres de manualidades en arcilla para elaborar collares y broches. La finalidad fue el aprendizaje colaborativo y creativo entre diferentes generaciones.

Probado el éxito de participación, los menores mostraron ser participativos, al igual que el alumnado de la Escuela-Taller. Los niños 


\section{Cambero \& Rangel-Aprendizaje Intergeneracional}

mostraban estar contentos por estos días compartidos con los jóvenes y mayores del pueblo. Estos últimos mostraron actitudes diferentes. Por un lado, un grupo de mayores de más edad fueron poco participativos, mientras que otro grupo, si disfrutaron mediante colaboraban con niños y jóvenes en un día diferente.

Con este programa se analizó si era necesario o no tener vínculos familiares para transmitir conceptos entre generaciones, y se concluyó, que no era necesario para lograr este objetivo de aprendizaje.

\section{Consolidación del Programa Intergeneracional}

Dado el éxito de la experiencia piloto el año anterior, en el curso 2017/2018 se celebró el mismo programa, aunque en esta ocasión no se contara con alumnado de la Escuela-Taller. Los participantes se agruparon en:

1. Alumnado de primero y segundo de Primaria del CEIP "Maestro Pedro Vera" con edades comprendidas entre 6 y 8 años. El alumnado estuvo acompañado por tutores.

2. Mayores de pisos tutelados gestionados por el Ayuntamiento de Oliva de la Frontera, más los usuarios del Centro de Día de la misma localidad. La cifra multiplicó la representación de este grupo etario con respecto al año anterior.

3. Alumnado del Taller de Empleo de "Atención socio sanitaria a personas dependientes en instituciones sociales", con edades superiores a 45 años, por lo que la generación y las enseñanzas fueron diferentes al año anterior. Tratándose de personas en preparación para el desarrollo de actividades de ocio geriátrico se involucraron en su organización.

Los objetivos fueron similares al año anterior, aunque en esta ocasión dentro de los hábitos saludables solo se fomentó el ejercicio físico-deportivo, adaptado a las necesidades de cada grupo etario.

Al ser más numeroso el número de participantes, las actividades se distribuyeron en tres grupos infantiles y dos para sénior, de modo que fueron rotando para participar en las siguientes actividades:

1. Taller de manualidad con temática de Semana Santa. Basada en la elaboración entre niños y mayores de "nazarenos" con materiales reutilizados, dirigida por el profesorado del colegio, que incentivaba que los ancianos relataran sus vivencias de niñez. Dado que los participantes de la 
Escuela-Taller eran mayores de 45 y menores que los residentes en los pisos tutelados, también se aprovechó para que contaran sus experiencias a los niños y así comparar sus correspondientes etapas infantiles y la evolución del pueblo.

2. Taller de juegos populares entre generaciones. Orientada por el maestro de Educación Física, para generar la curiosidad infantil y sénior a través de los juegos de ayer y hoy.

3. Taller de actividades acuáticas. Dirigida únicamente a los niños en la piscina climatizada, donde el socorrista y una maestra especialista organizaron actividades física-deportivas, así como hábitos saludables, a la vez que se les ofrecía esta actividad lúdica asociada al verano. También se les mostró a los niños los ejercicios acuáticos que los mayores ejercitaban.

En cuanto a la valoración de los distintos agentes y grupos etarios involucrados en estas actividades fueron similares a los descritos en los talleres del curso anterior.

\section{Discusión}

Tras el análisis de resultados de esta investigación sobre esta práctica intergeneracional a escala local, se confirman los beneficios individuales y colectivos del aprendizaje intergeneracional en contextos familiares y socioeducativos, con especial incidencia en la socialización infantil. La hipótesis queda verificada en cuanto a los resultados de aprendizaje de conceptos significativos en los niños, pero igualmente la promoción del envejecimiento activo de los adultos mayores que intervienen en estos procesos de transferencia de conocimiento, habilidades y valores.

En este momento histórico de diversidad generacional en familias y organizaciones, hay otro factor que no aparece en esta investigación relativo a la revolución tecnológica actual que marca el desarrollo de la sociedad de la información. Hay evidencias de como los grupos sociales, especialmente los que se encuentren en riesgo de exclusión digital como podrían ser las personas mayores, se incorporan activamente mediante su alfabetización tecnológica (Íñiguez-Berrozpe, Valero-Errazu y Elboj-Saso, 2018); incluso hay experiencias de espacios de aprendizaje integeneracional liderados por adultos mayores que practican el voluntariado sénior o cibervoluntariado en centros de mayores, escolares o penitenciarios (Cambero, 2015). 


\section{Cambero \& Rangel-Aprendizaje Intergeneracional}

Conforme al conocimiento científico consultado, esta investigación aporta empirismo sobre las influencias en el aprendizaje intergeneracional en los contextos analizados, ofreciendo resultados por un lado, sobre la tipología de conceptos adquiridos por niños, y por otro, la percepción de atención y utilidad social entre los mayores participantes en estas iniciativas socio-educativas. Además, se evidencia la disponibilidad de los distintos agentes comunitarios que intervienen en la organización y desarrollo de estos programas intergeneracionales, en este caso, en el medio rural extremeño. De ahí, la importancia de reforzar las pedagogías activas para la implementación de acciones educativas participativas social y etariamente, cuya finalidad sea el cambio de actitudes y conductas que prevengan el edadismo, como tercera forma de discriminación social en Europa, tras el racismo y el sexismo (Butler, 1980; Stallar, Decker y Bunnell, 2002).

La intergeneracionalidad es una constante a lo largo de la humanidad, acrecentada ante una coyuntura de envejecimiento demográfico que origina la coexistencia de personas de distintas cohortes de edad en familias, organizaciones y sociedad en general. Este hecho social obligará a cambios de mentalidad sobre la aceptación e integración de personas de diferentes generaciones, que suman más que resta en la convivencia cívica. De ahí que los programas intergeneracionales sean instrumentos de intervención sociocomunitario que permiten afrontar ciertos cambios demográficos, especialmente fomentando la participación activa de las personas mayores como ciudadanía sénior. Ese es el reto en la era de la longevidad, construir sociedades inclusivas y cohesionadas para todas las edades, que valoricen las contribuciones sénior dando como resultado la denominada huella generacional (Cambero, 2018).

Por otro lado, subrayar que estas prácticas intergeneracionales favorecen la cohesión social y territorial en el medio rural, y concretamente en Extremadura, donde al igual que en otras comunidades hay problemas de despoblación rural. Quizás los programas intergeneracionales sean un elemento estratégico más para lograr que los pueblos españoles sean lugares más atractivos para vivir, trabajar, estudiar y crecer en diversidad generacional, mediante la promoción del patrimonio natural, cultural y humano del medio rural. Esta sería una línea futura de investigación a desarrollar en el marco de la búsqueda de soluciones articuladas frente al reto demográfico en España. 


\section{Conclusiones}

Esta investigación ha permitido clasificar el conjunto de conceptos que los niños aprenden de los distintos miembros familiares que intervienen en su socialización. De los datos obtenidos, subrayar que los principales conceptos que aprenden estos menores de sus familiares adultos estarían englobados en la categoría de "Ética y moral", "Vivencias", "Educación medioambiental" y "Deportes"; mientras que los niños aprenden más de sus progenitores, seguidos de sus abuelos, y por último de sus hermanos. Una de las razones que explicaría este último dato sería que se les considera más compañeros de juegos que unos referentes para aprender.

En cuanto a las conclusiones, subrayar los siguientes aspectos:

1. Los niños aprenden principalmente de tres actores familiares. Primeramente, de padres y madres, seguidos de abuelos, y por último, de modo aislado de hermanos. Ninguno ha entendido que aprende conceptos de su núcleo familiar más extendido como tíos o primos. A la vez que se detecta a los actores participantes en la socialización infantil, tanto fuera como dentro del colegio.

2. Los conceptos se transmiten de modo espontáneo, ya que los temas que más se aprenden no son los más valorados en la transmisión de conocimiento, sino los que más les gusta tratar a los adultos, es decir, los temas relacionados con las vivencias personales. Sin embargo, otros conceptos enseñados como "Ética y moral" y "Educación ambiental" si son valorados en la transmisión de conocimientos y no se tienen en cuenta en conversaciones. Se entiende que estos conocimientos se enseñan por convicción más que por generación espontánea.

3. El alumnado se mostró satisfecho con la participación en los programas intergeneracionales. Se creó un ambiente lúdico mediante la utilización de recursos para el aprovechamiento del ocio y el tiempo libre infantil. Los niños mejoran sus habilidades sociales (empatía, tolerancia,...) y menor riesgo de comportamientos de riesgo social. Los docentes también expresaron satisfacción por los resultados de la experiencia, principalmente en el intercambio de experiencias de vida.

4. Por último, los mayores participantes en los programas intergeneracionales manifestaron agrado por unos días diferentes. Se fomentó la movilidad física y la psicomotricidad cuando ejercitaron la 
memoria mientras contaron vivencias de la niñez y aprendieron de los juegos infantiles actuales. El incremento en la actividad física, cognitiva y social, a partir de los programas intergeneracionales, puede ayudar a mejorar la salud para la población que envejece.

\section{Notas}

1 Se eligen estas edades como orientativas en los centros educativos analizados, pues el rango de edad de los docentes no es tan amplio. Pero con 23 años hacemos referencia a la edad mínima que un estudiante de Grado de Educación puede terminar sus estudios y con 65 años a la edad que suelen jubilarse.

\section{Referencias}

Butler, R. (1980). Ageism: A foreword. Journal of Social Issues, 36, 8-11. Caballero, M. y Baigorri, A. (2013). ¿Es operativo el concepto de generación? Aposta: Revista de ciencias sociales, 56. Recuperado de http://www.apostadigital.com/revistav3/hemeroteca/mcg1.pdf

Cambero, S. (2015). Ciudadanía y voluntariado sénior en la sociedad telemática. Estudio de AVIMEX como paradigma del envejecimiento activo e inclusivo en Extremadura (tesis doctoral). Universidad de Extremadura. Recuperado de http://dehesa.unex.es/xmlui/bitstream/handle/10662/3289/TDUEX_20 15_Cambero_Rivero.pdf?sequence $=1$

Cambero, S. (2018). Gestión de la edad en las ENL en Extremadura: la "huella generacional". Comunitania, International Journal of Social Work and Social Sciences, 16, pp. 81-101. http://dx.doi.org/10.5944/comunitania.16.2

Campo, C. (2004). La lengua extranjera como vehículo de una educación global. Tarbiya: Revista de investigación e innovación educativa, 35, 105-116. Recuperado de

https://www.uam.es/servicios/apoyodocencia/ice/tarbiya/pdf/revistas/ Tarbiya035.pdf

Campos, J. (1988). "Las habilidades atléticas: modelo para el aprendizaje de los fundamentos atléticos en el ámbito escolar" en La educación física en el curriculum de primaria, Villamón, M. (coord.), 224-269.

CENIE (2019). La diversión y el valor de los programas intergeneracionales. Centro Internacional sobre el Envejecimiento 
(CENIE). Recuperado de https://cenie.eu/en/node/324

Delors, J. (1996). "Los cuatro pilares de la educación” en La educación encierra un tesoro. Informe a la UNESCO de la Comisión internacional sobre la educación para el siglo XXI. Madrid, España: Santillana/UNESCO.

Fundación EDE (2015). Hacia una sociedad intergeneracional: ¿Cómo impulsar programas para todas las edades? Guía práctica. Centro del Conocimiento de Fundación EDE. Diputación Foral de Bizkaia.

Departamento de Acción Social.

Giró, J. (2006). Envejecimiento activo envejecimiento en positivo.

Universidad de la Rioja. Recuperado de https://dialnet.unirioja.es/servlet/libro?codigo $=343628$

Herranz, P. y Lacasa, P. (1989). Contexto y procesos cognitivos: La interacción niño-adulto, Infancia y Aprendizaje. Journal for the Study of Education and Development, 45, 25-48. Recuperado de https://dialnet.unirioja.es/descarga/articulo/48319.pdf

INE (2016). Estadística del Padrón continuo. Recuperado de

http://www.ine.es/dyngs/INEbase/es/categoria.htm?c=Estadistica_P\& cid $=1254734710990$

Iñiguez-Berrozpe, T., Valero-Errazu, D. y Elboj-Saso, C. (2018). Hacia una Sociedad de la Información inclusiva. Competencia tecnológica y habilidades relacionadas con las Tecnologías de la Información y la Comunicación (TIC) de los adultos maduros. Revista Mediterránea de Comunicación-Journal of Communication, 9(2), 25-40. doi:

10.14198/MEDCOM2018.9.2.9

López, S. (2001). La familia como contexto de aprendizaje de padres e hijos: límites, estructura y condiciones individuales. Bordón. Revista de pedagogía, 53, 1, 73-88.

Malrieu, P. (1979). El desarrollo intelectual: La curiosidad infantil. En JUIF, P., y LEIF, J. (coord.) Textos de psicología del niño y del adolescente. Narcea, 296-297.

Mestre, J., Guillén, J. y Caro, F. (2012). Abuelas cuidadoras en el siglo XXI: recurso de conciliación de la vida social y familiar. Portuaria, Revista de Trabajo Social, 12, 231-238. Recuperado de http://rabida.uhu.es/dspace/bitstream/handle/10272/5973/Abuelas_cui dadoras_en_el_siglo_XXI.pdf?sequence=2 


\section{Cambero \& Rangel-Aprendizaje Intergeneracional}

McCrea, J., Weissman, M. y Thorpe-Brown G. (2004). Connecting the generations: a practical guide for developing intergenerational programs. Pittsburgh, PA: Generations Together.

McKenna, A. (1980). El papel del adulto en el lenguaje del niño Infancia y Aprendizaje. Journal for the Study of Education and Development, 9, 84-94.

Moreno, E. (2001). Análisis de la influencia de la familia en los hábitos lectores de sus hijas e hijos: Un estudio etnográfico. Contextos educativos: Revista de educación, 4, 177-196.

Naciones Unidas (2002). Informe de la Segunda Asamblea Mundial sobre el Envejecimiento. Madrid, 2002. Ediciones Naciones Unidas, Nueva York, 5-48.

Prensky, M. (2001). Digital Natives, Digital Immigrants Part 1. On the Horizon, 9(5), 1-6. doi: 10.1108/10748120110424816

Quejereta, M. (2010). Sociedad, familia y aprendizaje: El papel de los contextos hogareños. Orientación y sociedad: Revista internacional e interdisciplinaria de orientación vocacional ocupacional, 10.

Recuperado de

http://www.memoria.fahce.unlp.edu.ar/art_revistas/pr.4759/pr.4759.p df

Robledo, P. (2012). Contexto familiar y aprendizaje escolar: estudios comparativos y de intervención (tesis Doctoral). Universidad de León. Recuperado de

https://buleria.unileon.es/bitstream/handle/10612/2255/tesis_f20eb5.P DF? sequence $=1$

Ruíz, J.M. (2000). Intervención logopédica en el ámbito escolar: el docente ante trastornos de aprendizaje de la lengua. Universidad Nacional de Educación a Distancia (UNED). Recuperado de http://espacio.uned.es/fez/eserv/bibliuned:editorial-Varia0137244PB01A01/Documento_0137244PB01A01.pdf

Sánchez, M. (2007). Programas intergeneracionales. Hacia una sociedad para todas las edades. Barcelona: Fundación "la Caixa".

Sánchez, M., Kaplan, M. y Sáez, J. (2010). Programas Intergeneracionales. Guía introductoria. Madrid: Instituto de Mayores y Servicios Sociales (IMSERSO).

Stallard, J.M., Decker, I.M. y Bunnell, J. (2002). Health Care for the Elderly: 
A Social Obligation. Nursing Forum, 37(2), 5-15.

Toribio, C. (2002). Monoparentalidad y solidaridad entre las mujeres en España: El papel de las abuelas cuando las hijas trabajan. Inguruak: Soziologia eta zientzi politikoaren euskal aldizkaria. Revista vasca de sociología y ciencia política, 34, 7-19.

Vera, J. (1987). La cultura próxima como fuente de contenidos significativos de un diseño curricular basado en la comprensión de conceptos. Teoría de la educación, 2, 35-44. Recuperado de http://campus.usal.es/ revistas_trabajo/index.php/11303743/article/viewFile/2871/2907

Santiago Cambero Rivero is a professor at the University of Extremadura, Spain

Noelia Rangel Preciado is a master graduate at the University of Extremadura, Spain

Contact Address: scamriv@unex.es 